\title{
Cancer Stem Cells and Cancer Stem Cell Inhibitors in Gastrointestinal Cancers
}

\author{
Joleen M Hubbard and Axel Grothey \\ Division of Medical Oncology, Mayo Clinic, Rochester, Minnesota, US
}

DOI: http://doi.org/10.17925/OHR.2016.12.01.41

\begin{abstract}
Cancer stem cells (CSCS) are a subpopulation of phenotypically distinct cancer cells that may play an important role in tumor pathogenesis. The gastrointestinal (GI) system provides a good example for investigation of the role of CSCs in tumor proliferation; GI CSCs are suitable for study due to their abundance, proliferative potential, and consistent structural arrangement that is maintained under tightly controlled signaling pathways. GI stem cells have a long lifespan and this, combined with their rapid turnover, may predispose them to forming CSCs. Alternative possible sources of GI CSCs include differentiated intestinal cells, bone marrow, and cancer cells. Therapies that specifically target CSCs present an exciting opportunity to treat patients with cancer. Enhanced understanding of CSC markers, such as CD133, CD44, and epithelial cell adhesion molecule (EPCAM), may facilitate development of therapies that target them. Among the stemness pathways that have been targeted are Wnt/ $\beta$-catenin, STAT, Notch, and Nanog.
\end{abstract}

\section{Keywords}

Cancer therapy, cancer stem cells, CD133, CD44, epithelial cell adhesion molecule (EpCAM), focal adhesion kinase (FAK), gastrointestinal tumor, Hedgehog, Nanog, Notch, STAT3, Wnt/ $\beta$-catenin

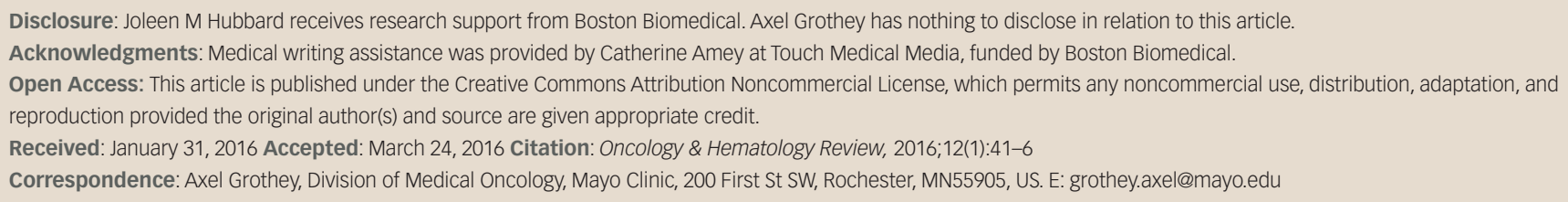

Support: The publication of this article was supported by Boston Biomedical, who were given the opportunity to review the article for scientific accuracy before submission. Any resulting changes were made at the author's/authors' discretion. The publication of this article was supported by Boston Biomedical. The views and opinions expressed are those of the author and do not necessarily reflect those of Boston Biomedical.

As is the case with most solid tumors, gastrointestinal (GI) tumors are treated in a variety of modalities, which are used singularly or in combination, including surgery, targeted therapies, radiation, and chemotherapy. Survival rates by stage in colon and rectal cancer are given in Table 1.

Cancer stem cells (CSCS) are believed to be malignant cells that have the capacity to initiate and maintain tumor growth and survival. ${ }^{1}$ They are more resistant to radiation and chemotherapeutic agents than other cancer cells. ${ }^{2}$ The presence of CSCs following cancer therapy may explain the initiation of metastasis and later recurrence of cancers, which can occur even when there is a good initial response to radiation or chemotherapy. ${ }^{3}$ The first experiments suggesting the presence of CSCs in Gl cancers were conducted in 2007.,5 The investigators used flow cytometry to isolate CSCs using CD133 as a marker and then demonstrated the ability of these CD133-positive cells to form xenografts in non-obese diabetic/severe combined immunodeficiency mice.

\section{Origin of cancer stem cells of the gastrointestinal system}

A schematic representation of an individual colon crypt is depicted in Figure 1. Stem cells lie at the bottom of the crypt and through asymmetric division are responsible for generating all epithelial cell types along the crypt-villus axis. GI stem cells may be intrinsically prone to forming CSCs because of their long lifespan combined with rapid turnover and it is widely believed that CSCs are derived from normal stem cells. ${ }^{6,7}$ Other possible sources for GI CSCs include dedifferentiated intestinal cells, possibly via nuclear factor-kappa-B (NF-кB) modulation of Wnt signaling7,8 and bone marrow-derived progenitor cells progressing through metaplasia and dysplasia to cancer. ${ }^{9}$

\section{Cancer stem cell biomarkers CD133}

The CD133 molecule, which is also known as prominin-1, is a pentaspantransmembrane glycoprotein that has been shown to be located mainly in membrane protrusions. ${ }^{10}$ It was first identified as a surface protein marker of a subset of hematopoietic stem and progenitor cells as early as 1997,11 but its biologic function has yet to be elucidated. In 2007, CD133-positive cells separated from colorectal tumor cells were demonstrated to possess self-renewal properties and high tumorigenic potential. ${ }^{4,5}$ Systematic reviews have indicated that $\mathrm{CD} 133$ is a prognostic factor in colorectal cancer (CRC) ${ }^{12}$ and gastric cancer. ${ }^{13}$ Carbon nanotube-conjugated CD133- 
Table 1: Survival rates for (A) colon cancer and (B) rectal cancer by stage

\begin{tabular}{ll} 
A & Five-year Relative Survival Rate \\
Stage & $92 \%$ \\
\hline IIA & $87 \%$ \\
IIB & $63 \%$ \\
IIIA & $89 \%$ \\
IIIB & $69 \%$ \\
IIIC & $53 \%$ \\
IV & $11 \%$ \\
B & Five-year Relative Survival Rate \\
Stage & $87 \%$ \\
I & $80 \%$ \\
IIA & $49 \%$ \\
IIB & $84 \%$ \\
IIIA & $71 \%$ \\
IIIB & $58 \%$ \\
IIIC & $12 \%$ \\
IV &
\end{tabular}

Source: National Cancer Institute's Surveillance, Epidemiology, and End Results (SEER) database, looking at people diagnosed with colon and rectal cancer between 2004 and 2010. These statistics are based on a previous version of the staging system. In that version, there was no stage IIC (those cancers were considered stage IIB). Also, some cancers that are now considered stage IIIC were classified as stage IIIB, while some other cancers that are now considered stage IIIB were classified as stage IIIC.

Figure 1: The possible origins of cancer stem cells of the gastrointestinal system

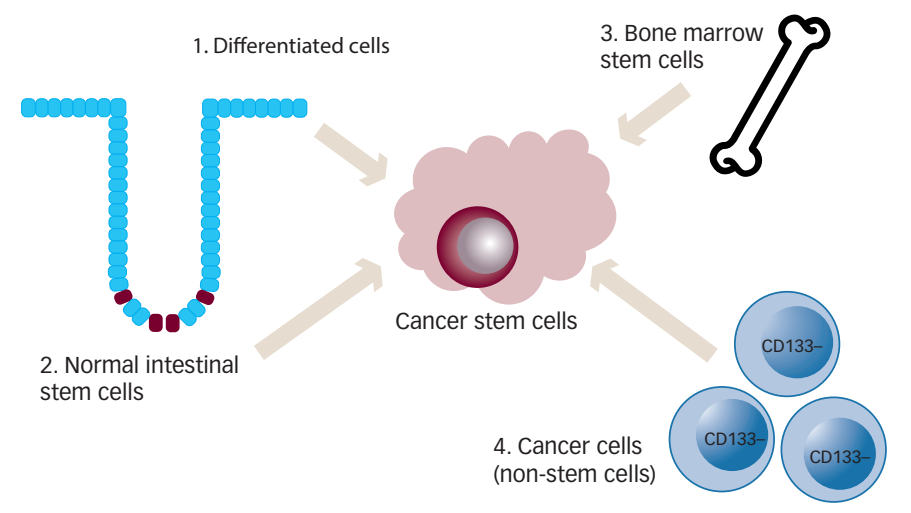

positive monoclonal antibodies led to photothemolysis of CD133-positive glioblastoma cells in vivo and in mice, ${ }^{14}$ suggesting that CD133 may represent a useful target to selectively inhibit CSCs in CD133-expressing tumor types.

\section{CD44}

CD44, a cell surface adhesion molecule, is the principal receptor for hyaluronate, which is the most abundant extracellular matrix component. ${ }^{15,16}$ CD44 has been suggested to perform functions in CSCs such as mediation of adhesion and homing to the stem cell niche, enhancement of antiapoptotic proteins and surface efflux pump expression, regulation of the cellular redox status and the response to the activation of the canonical Wnt pathway. ${ }^{17-19}$ Future research is needed to elucidate the suitability of CD44 as a CSC marker in GI cancer and its role in tumorigenesis.

\section{EpCAM}

Epithelial cell adhesion molecule (EPCAM), initially described in human CRC as a tumor-associated antigen ${ }^{20}$ is expressed highly in a range of human epithelial normal and cancer tissues, including the colon. ${ }^{21}$ Several lines of evidence indicate that EPCAM is involved in cell adhesion, proliferation, migration, and cancer and stem cell signaling. ${ }^{22,23}$

\section{Other potential markers}

More recently, identified possible markers of CSCs include: CD29/integrin $\beta 1$, a mucin-like cell adhesion molecule, ${ }^{24}$ CD24/HSA, a extracellular matrix protein receptor that is involved in regulation of cell migration, proliferation, survival, differentiation, and death;25 Lgr5/Gpr49, a receptor for R-spondin proteins, ${ }^{26}$ and CD166/ALCAM, a cell adhesion molecule. ${ }^{27}$

\section{Limitations of cancer stem cell markers}

Identification and isolation of CSCs using putative surface markers has received much attention in cancer research. However, heterogeneity among GI tumors and GI tumor subtypes has led to difficulty in pinpointing unique markers. Expression of surface markers varies at different tumor stages and their main regulatory functions are not understood fully. ${ }^{28}$ Lack of universal expression of surface markers has obfuscated their use and no optimal combination of markers has been confirmed for the identification of CSCs. Further, non-CSCs have been shown to also express some of these markers. ${ }^{29}$ CSC markers are an area of continuous development as more studies identify molecules that may serve as new CSC markers and help to identify CSCs in Gl cancers in a tissue-specific manner.

\section{Cancer stemness and epithelial-to-mesenchymal transition}

Stemness, initially taken to mean expression of stem cell genes, such as Nanog, Oct4, and Sox2, is a defining property of embryonic and adult stem cells. ${ }^{30}$ Stemness can be measured by a cell's ability to form spheres when cultured in stem cell media. ${ }^{31}$ Chemotherapy ${ }^{32}$ and radiation ${ }^{33}$ have been found to induce the expression of stemness genes in cancer cells in vitro, thereby enriching the CSC population in the residual tumor.

Epithelial-to-mesenchymal transition (EMT) is the capacity of epithelial cells to acquire mesenchymal traits to allow local invasion into surrounding tissues and systemic dissemination to distant organ sites. ${ }^{3}$ Recent evidence indicates that EMT can induce differentiated cancer cells into a CSC-like state, suggesting a functional link between stemness and EMT. ${ }^{34}$

\section{Stemness pathways associated with cancer stem cells in gastrointestinal malignancies- therapeutic targets}

The desired aim is to overcome resistance to chemotherapeutics and reduce therapy-related toxicity by developing treatments that are specific for CSCs and that are not toxic to healthy tissues. Examples of stemness pathways as therapeutic targets are discussed below.

\section{Nanog}

Nanog, an essential regulator of embryonic stem cell self-renewal that inhibits differentiation, is overexpressed in a variety of cancers including 
Table 2: Upstream regulators $(A)$ and downstream modulators (B) of Nanog 91

\section{A}

Upstream Regulators of Nanog

\begin{tabular}{lll} 
Targets & Mediated Cell Functions & References \\
STAT3 & Maintenance of pluripotency & $4,93,95$ \\
Ezh2 & Epigenetic regulation of Nanog, self-renewal & 72 \\
Hedgehog & Self-renewal, tumorigenicity & $32,71,89$ \\
\hline TLR4 & Tumor formation & 132 \\
p53 & Differentiation, CSC properties acquirement & $32,84,85$ \\
ESrrb & Maintaining Nanog expression & 111,112 \\
HIF2 $\alpha$ & Enhances Nanog and CSC properties under & 133 \\
& hypoxia & \\
PI3K/Akt & Tumorigenesis, cell survival, and selfrenewal & $33,115,121,134$
\end{tabular}

AKt $=$ protein kinase $B ; C S C=$ cancer stem cells; Esrrb = estrogen-related receptor $\beta$; Ezh2 = enhancer of zeste homolog 2; HIF2 $\alpha=$ hypoxia inducible factor $2 \alpha ; P I 3 K=$

phosphatidylinositide 3-kinase; STAT3 = signal transducer and activator of transcription 3; TLR4 = toll-like receptor 4 .

B

Downstream Modulators of Nanog

\begin{tabular}{lll} 
Targets & Mediated Cell Functions & References \\
Cyclin D1 & $\begin{array}{l}\text { Cell cycle progression, G0/G1 arrest, and } \\
\text { proliferation }\end{array}$ & 76,77 \\
\hline ABCB1 & Chemoresistance & $34,107-109$ \\
\hline GDF3 & Growth and differentiation & 105 \\
\hline Dnmt1 & Maintenance of self-renewal and undifferentiated135 \\
& state & 78 \\
\hline E-cadherin & Migration and invasion & 78 \\
FoxJ1 & Migration and invasion & 79 \\
\hline TCl1a & Immune evasion & 124 \\
\hline DKk1 & Paracrine communication with stroma & 84,122 \\
FAK & CSC proliferation and motility, tumor survival
\end{tabular}

$\overline{A B C B 1}=A T P$-binding cassette sub-family $B$ member $1 ; C S C$ = cancer stem cell; DKK1 = dickkopf-related protein 1; Dnmt1 = DNA (cytosine-5)-methyltransferase 1; FAK = focal adhesion kinase; FoxJ1 = forkhead box protein J1; GDF3 = growth differentiation factor-3; $T c / 1 a=$ T-cell leukemia/lymphoma protein $1 \mathrm{~A}$.

those of the GI system ${ }^{35,36}$ High levels of Nanog expression are associated with advanced stages of cancer and a poor prognosis, suggesting that it may play a key role in tumor transformation, tumorigenesis, and tumor metastasis. ${ }^{35}$ Nanog is involved in a complex regulatory network that determines cell fate, proliferation, and apoptosis (Table 2). It is therefore a promising therapeutic target. Genetic ablation of Nanog in SW620 colorectal carcinoma cells suppressed both tumor growth in athymic nude mice and cell proliferation in vitro. ${ }^{37}$ Further, ablation mediated by short hairpin RNA decreased the expression of core CSC transcription factors, supporting a role for Nanog as a signali ng hub in CSCs. ${ }^{38}$

BBI503 is an orally administered investigational agent designed to inhibit Nanog and other CSC pathways by targeting kinases. Early signs of antitumor activity have been observed in pre-treated patients with advanced cancer (BBI503-101, NCT01781455). ${ }^{39}$ In this ongoing, first-in-human, open-label phase I dose-escalation study, 11 of 20 evaluable patients (55\%) had stable disease with a median time to progression of 16 weeks. of these 11 patients, tumor regression and/or prolonged stable disease
Figure 2: STAT3 activation in cell proliferation, cell survival, invasion, angiogenesis, and metastasis ${ }^{41}$

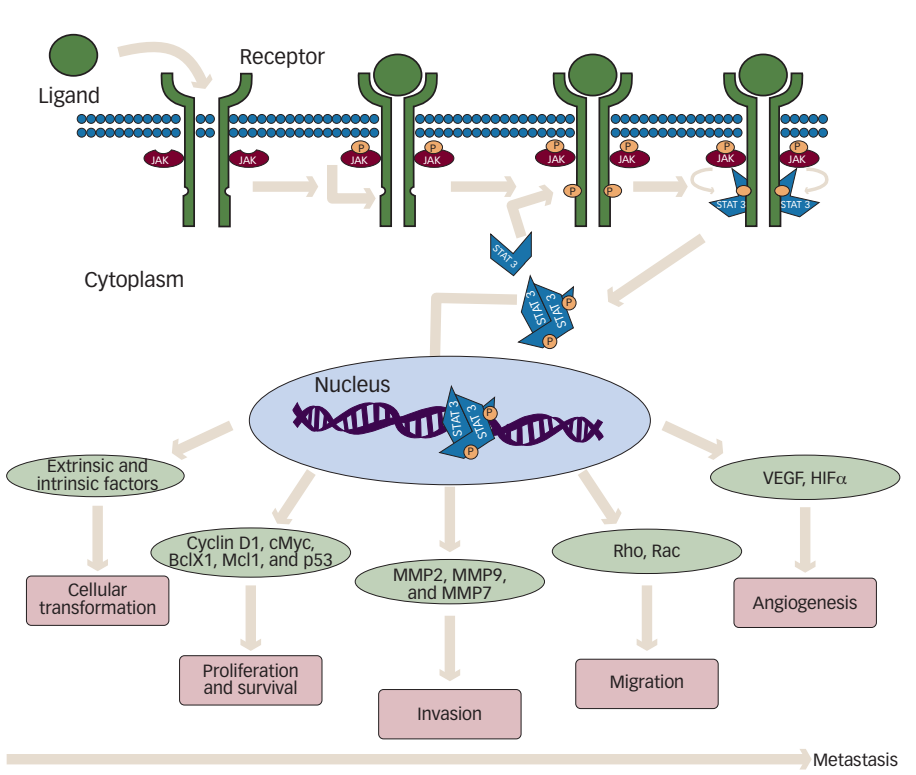

Reproduced from Kamran et al. ${ }^{41}$

( $\geq 16$ weeks) were observed in 10 patients (50\% all enrolled patients). Further, in biopsied tumor tissues, dose-dependent pharmacodynamics effects of decreased expression level of Nanog were observed. The recommended phase II dose of continuous once daily BBI503 was 300 $\mathrm{mg} /$ day. At this dose, BBI503 was shown to be well tolerated and the pharmacokinetic profile supports once-daily dosing as an acceptable administration schedule. Mild GI adverse events were observed, including grade 1 and 2 diarrhea, adnominal cramps, nausea, and anorexia. Grade 3 diarrhea was noted in two patients at $450 \mathrm{mg}$ once daily.

In an extension study of BBI503-101, BBI503 was administered to patients with advanced $\mathrm{CRC} .{ }^{40}$ Patients $(\mathrm{n}=47$ ) with heavily pre-treated CRC were enrolled. The disease control rate in evaluable patients $(n=39)$ with high Nanog expression (biomarker positive) was $56 \%$, whereas in biomarkernegative patients, the disease control rate was $13 \%(p=0.04)$. Median overall survival in biomarker-positive patients (intent-to-treat) was 38 weeks compared with 16 weeks in biomarker-negative patients ( $p=0.089$ Log-Rank). BBI503 was well tolerated at the recommended phase II dose of $300 \mathrm{mg}$ once daily; grade 3 adverse events were diarrhea $(n=5)$, fatigue $(n=4)$, nausea $(n=1)$, and weight loss $(n=1)$.

\section{STAT3}

STAT3 is a latent cytoplasmic transcription factor that appears to be activated constitutively in many cancers, to play a pivotal role in metastasis and tumor growth, and is associated with decreased survival. ${ }^{41-43}$ It was first discovered in 1994 as a signal transducer from cell surface receptors to the nucleus. ${ }^{44}$ STAT activation has been suggested to be important at every stage of metastasis (Figure 2).

Napabucasin (BBI608) is an orally administered investigational agent that has been designed to inhibit CSC pathways including STAT3, Nanog, and $\beta$-catenin pathways by targeting STAT3.41,45 Pre-clinical data indicate that BBI608 inhibits STAT3-driven transcription and suppresses metastasis 
Figure 3: Notch in Tumor Metastasis as an Epithelialto-mesenchymal Transition Inducer ${ }^{56}$

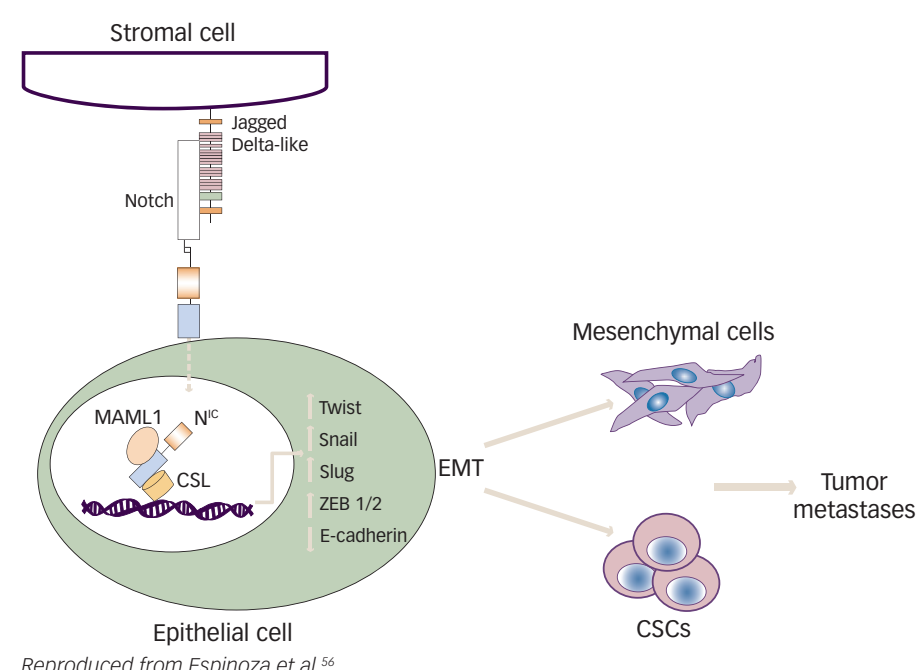

Reproduced from Espinoza et al. ${ }^{56}$

\section{Figure 4: Regulation of $\beta$-catenin Protein by Notch ${ }^{61}$}

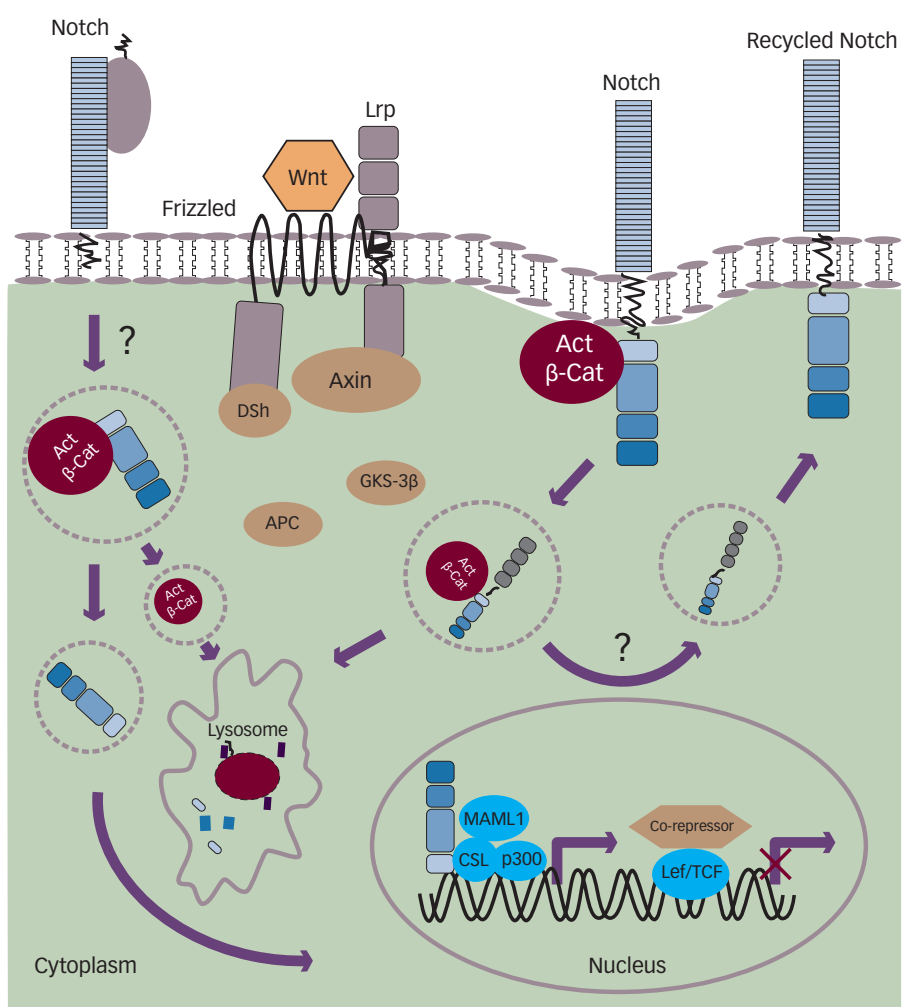

Post-Translational Regulation of $\beta$-Catenin Protein by Notch. Notch can negatively regulate active $\beta$-catenin levels an a non-canonical fashion. In the presence of Wnts., membrane-bound Notch forms a complex with active $\beta$-catenin and degrades active $\beta$-catenin through an endoIysosomal pathway. The degradation is independent of GSK3ß-dependent destruction complex. Whether Notch is recycled back to the membrane is unclear. Notch intracellular domain (NICD) can also regulate active $\beta$-catenin levels in a similar mechanism but it is unknown whether endogenously processed NICD regulates active $\beta$-catenin protein. Protein interaction can be either direct or indirect. Reproduced with permission from Anderson et al. ${ }^{61}$

and cancer relapse. ${ }^{45,46} \mathrm{An}$ ongoing phase Ib study has demonstrated that BBI608 and weekly paclitaxel can be combined at full dose in patients with advanced malignancies (NCT01325441). ${ }^{47}$ Early anti-tumor activity was shown across several tumor types, especially in those patients with gastric and gastroesophageal junction adenocarcinoma. BBI608-201 (NCT 01325441) is a phase Ib/II study of BBI608 combined with paclitaxel in advanced gastric and gastroesophageal junction adenocarcinoma.48 BBI608 at doses of $480 \mathrm{mg}$ twice daily (BID) and $500 \mathrm{mg}$ BID plus weekly paclitaxel $\left(80 \mathrm{mg} / \mathrm{m}^{2}\right)$ was well tolerated. Common adverse events included grade 1 to 2 diarrhea, abdominal cramping, nausea, and vomiting. Grade 3 adverse events including vomiting, diarrhea of five days' duration or longer, fatigue, abdominal cramps, nausea, and dehydration. Early signs of anti-cancer activity were observed in a cohort of heavily pretreated patients. The BRIGHTER trial is a phase III randomized, doubleblind, placebo-controlled clinical trial of BBI608 plus weekly paclitaxel versus placebo plus weekly paclitaxel in adult patients with advanced, previously treated gastric and gastroesophageal junction adenocarcinoma (NCT02178956). ${ }^{49}$ There is also early indication in BBI608-246, that BBI608 can be administered with folinic acid, 5-flurouracil (5-FU) and irinotecan (FOLFIRI) with and without bevacizumab in patients with advanced CRC (NCT02024607),50 and in BBI608-224 with panitumumab in KRAS wild-type patients with metastatic CRC (NCT01776307). ${ }^{51}$ Early signs of anti-cancer activity in these studies warrant further investigation of BBI608. A phase III randomized study of BBI608 plus best supportive care versus placebo plus best supportive care is being assessed in patients with pre-treated advanced colorectal carcinoma (NCT01830621). ${ }^{52}$

\section{Notch}

Notch plays a role in embryogenesis, cellular homeostasis, differentiation, EMT, and apoptosis (Figure 3). ${ }^{53-56}$ Notch signaling is initiated by ligand binding to the Notch receptor, which then undergoes a two-step proteolytic cleavage by a disintegrin and metalloproteases family proteases and $\gamma$-secretase. ${ }^{57}$ Notch signaling can be inhibited by two major classes of Notch inhibitors: $\gamma$-secretase inhibitors (GSIs), including R04929097, MRK-003, MK-0752, and PF03084014, and monoclonal antibodies directing against Notch receptors or ligands. GSIs have been shown to provide clinical benefit; for example, PF0308414 demonstrated early anti-tumor activity in a phase I dose-finding study in patients with advanced-stage solid tumors. ${ }^{58}$ A phase II study of 37 patients with metastatic CRC who had received at least two prior lines of therapy in the metastatic setting found that RO4929097 monotherapy did not demonstrate significant drug activity. ${ }^{59}$

Notch mediates biologic process through: a canonical pathway (involving ligand-induced cleavage of Notch for transcriptional regulation that includes Notch 1-4 and five Notch ligands Delta like, 1, 3 and 4 and Jagged 1 and 2;60 and non-canonical pathways. ${ }^{55,61}$ Notch has also been implicated in crosstalk with other oncogenic pathways such as Hedgehog and Wnt signaling. ${ }^{62}$ Phase I/II trials of Notch inhibitors in solid tumors are in progress (NCT01647828, NCT01859741, NCT01277146, NCT01957007, NCT02259582, NCT01952249, NCT01189929, NCT01952249, NCT01189929). Examples of Notch inhibitors include the compounds tarextumab (OMP-59R5) and demcizumab (OMP21M18). OMP-59R5, which blocks both Notch2 and Notch3 signaling, has demonstrated any anti-tumor effect in patient-derived xenograft tumors. ${ }^{63}$ A phase I trial $(n=55)$ of OMP-21M18, which targets Notch ligand Delta like 4, revealed anti-tumor activity and good tolerability at doses of $\leq 5 \mathrm{mg}$ weekly. ${ }^{64}$

\section{Wnt/ $\beta$-catenin}

Notch signaling can also interact with Wnt/ $\beta$-catenin signaling (Figure 4). ${ }^{55,61}$ The Wnt pathway has a key role in embryogenesis, with effects that 
regulate proliferation and apoptosis in developing cells. ${ }^{65}$ Wnt signaling disruptions are observed in a variety of $\mathrm{Gl}$ cancers ${ }^{66}$ and may have a role in inducing EMT. ${ }^{67}$ Trials involving Wnt/ $\beta$-catenin inhibitors are underway, including BBI608 in gastric and gastroesophageal junction cancer (NCT02178956) and in advanced CRC (NCT01776307) as discussed in the STAT3 section. BBI608 has demonstrated early anticancer activity in a phase I trial in patients with CRC and anal squamous carcinoma. ${ }^{68}$

Monoclonal antibodies, which inhibit Wnt signaling either by neutralizing Wht ligands or by inhibiting Wnt receptors Frizzled (FZ) and LRP, are also under clinical investigation. Vantictumab (OMP-18R5), a monoclonal antibody that binds five Fz receptors and inhibits Wht signaling, and a fusion protein decoy receptor, ipafricept (OMP-54F28), are under study in phase I studies in advanced stage solid tumors (ClinicalTrials. gov NCT01345201, NCT02005315, NCT01957007, NCT01973309, NCT01608867, NCT02069145, NCT02092363). OMP-54F28 has been demonstrated to inhibit patient-derived xenograft tumor growth and to decrease CSC numbers. ${ }^{69}$

\section{Additional signaling pathways}

There are many other signaling pathways currently under investigation. Focal adhesion kinase (FAK) is a non-receptor tyrosine kinase ${ }^{70}$ with important roles in adhesion, survival, motility, metastasis, angiogenesis, lymphangiogenesis, cancer stem functions, tumor microenvironment, and EMT. ${ }^{71-76}$ Current FAK inhibitors mostly target the FAK kinase domain with the ATP-binding site to inhibit FAK kinase activity.7 Transforming growth factor- $\beta$ signaling is important for self-renewal and maintenance in the formation of GI cancers.78,79 Hedgehog describes a complex of molecules that regulate cell differentiation, regeneration, and stem cell properties. ${ }^{80} \mathrm{It}$ is central to the development and homeostasis of gut tissue and is deregulated in GI cancers. ${ }^{81}$ The stemness factor Nanog is one of the major targets for Hedgehog signaling. ${ }^{82}$ Specific inhibitors of Hedgehog signaling, such as vismodegib (GDC-0449), and sonidegib (LDE225) are being examined in clinical trials in addition to the approved indication for basal cell carcinoma. ${ }^{83}$ Addition of vismodegib to oxaliplatin with 5-FU and folinic acid (FOLFOX) chemotherapy did not improve progression-free survival in patients with gastric and gastroesophageal junction cancer. ${ }^{84}$

Phosphatase and tensin homolog (PTEN) is a phosphatase that antagonizes the activity of PI3 kinase; PTEN-deficient mice demonstrate an increase in intestinal stem cells, which results in excess crypt formation. ${ }^{85}$ The PTEN pathway helps regulate the proliferative rate and number of intestinal stem cells.

\section{Induction of cellular quiescence}

An alternative to the CSC inhibition discussed involves the chemical induction of cellular quiescence. This is a state of reversible cell cycle arrest, associated with reduced translation rate, activation of autophagy, and a low metabolic rate as characterized by decreased glycolysis. ${ }^{86}$ This is a burgeoning area of research and it has been suggested that the efficacy of CSC inhibitors and conventional therapies may be enhanced if used in combination with chemoquiescence-inducing agents such as chloroquine and its analogues. ${ }^{87-89}$

\section{Cancer stem cell inhibitor trial design}

Given that the cSCs constitute only a small proportion of an established tumor, a response rate read-out might not be the most suitable endpoint for a CSC inhibitor trial. The effect could be delayed and may better be captured with time-related endpoints using response criteria developed for immunotherapies. ${ }^{90}$

\section{Conclusion}

The discovery of CSCs-which have been described in GI neoplasms such as colon, pancreas, liver, and gastroesophageal tumors_-provides promise of a suitable target for improving future oncological treatment. CSCs of the GI system are well suited to research as they are abundant, and have proliferative potential, as well as a uniform structural arrangement which is maintained under tightly controlled signaling pathways. The CSC model has been criticized for failing to take into account the heterogeneous nature of Gl cancers. However, CSCs themselves may evolve over time, giving rise to cells that are both genetically and functionally heterogeneous. ${ }^{29}$ Accurate targeting of CSCs must be preceded by precise identification and characterization of those cells. More research is therefore needed to determine which and how many markers need to be considered in the identification of GI CSCS.

CSC-targeted therapies may represent a new treatment modality in patients with cancer. Research continues to target CSCs to render them more chemo- and radio-sensitive, inhibit their potential to proliferate and undergo EMT, thus decreasing the incidence of metastases. Future challenges include optimizing the study design for determining investigational agents' efficacy in cancer.
1. Clarke MF, Dick JE, Dirks PB, et al., Cancer stem cells-perspectives on current status and future directions: AACR Workshop on cancer stem cells, Cancer Res, 2006;66:9339-44.

2. Clevers $H$, The cancer stem cell: premises, promises and challenges, Nat Med, 2011;17:313-9.

3. Sampieri K, Fodde R, Cancer stem cells and metastasis, Semin Cancer Biol, 2012;22:187-93.

4. Ricci-Vitiani L, Lombardi DG, Pilozzi E, et al., Identification and expansion of human colon-cancer-initiating cells, Nature 2007:445:111-5.

5. O'Brien CA, Pollett A, Gallinger S, Dick JE, A human colon cancer cell capable of initiating tumour growth in immunodeficient mice, Nature, 2007;445:106-10.

6. Heath JP, Epithelial cell migration in the intestine, Cell Biol Int, 1996;20:139-46.

7. Shih IM, Wang TL, Traverso G, et al., Top-down morphogenesis of colorectal tumors, Proc Natl Acad Sci U S A, 2001:98:2640-5.

8. Schwitalla S, Fingerle AA, Cammareri P et al., Intestinal tumorigenesis initiated by dedifferentiation and acquisition of stem-cell-like properties, Cell, 2013;152:25-38.

9. Houghton J, Stoicov C, Nomura S, et al., Gastric cancer originating from bone marrow-derived cells, Science (New York, NY) 2004:306:1568-71.

10. Corbeil D, Roper K, Fargeas CA, et al., Prominin: a story of cholesterol, plasma membrane protrusions and human pathology, Traffic, 2001;2:82-91.

11. Miraglia S, Godfrey W, Yin AH, et al., A novel five-transmembrane hematopoietic stem cell antigen: isolation, characterization, and molecular cloning, Blood, 1997:90:5013-21.

12. Chen S, Song $X$, Chen $Z$ et al, CD133 expression and the prognosis of colorectal cancer: a systematic review and metaanalysis, PloS one, 2013;8:e56380.

13. Wen L, Chen $X Z$, Yang $K$, et al., Prognostic value of cancer stem cell marker CD133 expression in gastric cancer: a systematic review, PloS one, 2013;8:e59154.

14. Wang $\mathrm{CH}$, Chiou $\mathrm{SH}$, Chou $\mathrm{CP}$, et al., Photothermolysis of glioblastoma stem-like cells targeted by carbon nanotubes conjugated with CD133 monoclonal antibody Nanomedicine, 2011:7:69-79.

15. Aruffo A, Stamenkovic I, Melnick M, et al., CD44 is the principal cell surface receptor for hyaluronate, Cell, 1990;61:1303-13.

16. Almond A, Hyaluronan, Cell Mol Life SCi, 2007;64:1591-6.

17. Wielenga VJ, Smits R, Korinek V, et al., Expression of CD44 in Apc and Tcf mutant mice implies regulation by the WNT pathway, Am J Pathol, 1999:154:515-23.

18. Hao J, Chen $\mathrm{H}$, Madigan MC, et al., Co-expression of CD147 (EMMPRIN), CD44V3-10, MDR1 and monocarboxylate transporters is associated with prostate cancer drug resistance and progression, Br J Cancer, 2010;103:1008-18.

19. Ishimoto T, Nagano O, Yae T, et al., CD44 variant regulates redox status in cancer cells by stabilizing the XCT subunit of system $\times C(-)$ and thereby promotes tumor growth, Cancer Cell, system XC(-) and ther

20. Herlyn M, Steplewski Z, Herlyn D, Koprowski H, Colorectal carcinoma-specific antigen: detection by means of monoclonal antibodies, Proc Natl Acad Sci U S A, 1979;76:1438-42.

21. Went $P$, Vasei $M$, Bubendorf $L$, et al., Frequent high-level expression of the immunotherapeutic target Ep-CAM in colon, stomach, prostate and lung cancers, $\mathrm{Br} \perp \mathrm{Cancer}$ 2006:94:128-35

22. Trzpis M, MCLaughlin PM, de Leij LM, Harmsen MC, Epithelial cell adhesion molecule: more than a carcinoma marker and adhesion molecule, Am J Pathol, 2007;171:386-95.

23. Denzel S, Maetzel D, Mack B, et al., Initial activation of EpCAM 
cleavage via cell-to-cell contact, BMC Cancer, 2009;9:402.

24. Brizzi MF, Tarone G, Defilippi P, Extracellular matrix, integrins, and growth factors as tailors of the stem cell niche, Curr Opin Cell Biol, 2012:24:645-51.

25. Kristiansen $G$, Sammar M, Altevogt P, Tumour biological aspects of CD24, a mucin-like adhesion molecule, J Mol Histol, 2004;35:255-62

26. de Lau WB, Snel B, Clevers HC, The R-spondin protein family, Genome Biol, 2012;13:242

27. Lehmann JM, Riethmuller G, Johnson JP, MUC18, a marker of tumor progression in human melanoma, shows sequence similarity to the neural cell adhesion molecules of the immunoglobulin superfamily, Proc Natl Acad Sci U S A, 1989;86:9891-5.

28. Jaggupilli A, Elkord E, Significance of CD44 and CD24 as cancer stem cell markers: an enduring ambiguity, Clin Dev Immunol, 2012;2012:708036

29. Chen K, Huang $\mathrm{YH}$, Chen JL, Understanding and targeting cancer stem cells: therapeutic implications and challenges, Acta Pharmacol Sin, 2013;34:732-40.

30. Reya T, Morrison SJ, Clarke MF, Weissman IL, Stem cells, cancer, and cancer stem cells, Nature, 2001:414:105-11

31. Chen SF, Chang YC, Nieh S, et al., Nonadhesive culture system as a model of rapid sphere formation with cancer stem cell properties, PloS one, 2012;7:e31864.

32. Hu X, Ghisolfi L, Keates AC, et al., Induction of cancer cell stemness by chemotherapy, Cell Cycle, 2012;11:2691-8.

33. Ghisolfi L, Keates AC, Hu X, et al., Ionizing radiation induces stemness in cancer cells, PloS one, 2012;7:e43628.

34. Mani SA, Guo W, Liao MJ, et al., The epithelial-mesenchyma transition generates cells with properties of stem cells, Cell, 2008:133:704-15

35. Sun AX, Liu CJ, Sun ZQ, Wei Z, NANOG: a promising target for digestive malignant tumors, World I Gastroenterol, 2014;20:13071-8

36. Chambers I, Colby D, Robertson M, et al., Functional expression cloning of Nanog, a pluripotency sustaining factor in embryonic stem cells, Cell, 2003:113:643-55.

37. Ishiguro $\mathrm{T}$, Sato $\mathrm{A}$, Ohata $\mathrm{H}$, et al., Differential expression of nanog1 and nanogp8 in colon cancer cells, Biochem Biophys Res Commun, 2012;418:199-204.

38. Zhang J, Espinoza LA, Kinders RJ, et al., NANOG modulates stemness in human colorectal cancer, Oncogene, 2013;32:4397-405

39. Laurie SA, Jonker DJ. Edenfield WJ, et al., A phase 1 doseescalation study of BBI503, a first-in-class cancer stemness kinase inhibitor in adult patients with advanced solid tumors. 2014 American Society of Clinical Oncology (ASCO) Annual Meeting; Chicago, IL, USA, 2014

40. Jonker D, Laurie S, Cote G, et al., BBI503-101: Phase 1 Extension Study of BBI503, a First-in-Class Cancer Stemness Kinase Inhibitor, in Patients with Advanced Colorectal Cancer. The 2015 American Society of Clinical Oncology (ASCO) Annual Meeting. 29 May-2 June, 2015: Chicago, IL, USA, 2015

41. Kamran MZ, Patil P, Gude RP, Role of STAT3 in cancer metastasis and translational advances, Biomed Res int, 2013:2013:421821.

42. Kim DY, Cha ST, Ahn DH, et al., STAT3 expression in gastric cancer indicates a poor prognosis, J Gastroenterol Hepatol, 2009;24:646-51

43. Yakata Y, Nakayama T, Yoshizaki A, et al., Expression of p-STAT3 in human gastric carcinoma: significant correlation in tumour invasion and prognosis, Int I Oncol, 2007:30:437-42.

44. Akira $S$, Nishio $Y$, Inoue $M$ et al, Molecular cloning of APRE, a novel IFN-stimulated gene factor 3 p91-related transcription factor involved in the gp130-mediated signaling pathway, Cell, 1994:77:63-71.

45. Li Y, Rogoff HA, Keates S, et al., Suppression of cancer relapse and metastasis by inhibiting cancer stemness, Proc Natl Acad SCi U S A, 2015;112:1839-44

46. Li Y, Rogoff HA, Keates S, et al., Inhibition of stemness by BBI608 is sufficient to suppress cancer relapse and metastasis (abstract number: LB-253). The American Association for Cancer Research (AACR) 2015; 18-22 April, 2015: Philadelphia, PA, 2015.

47. Hitron M, Stephenson J, Kim N, et al., A phase $1 \mathrm{~b}$ study of the cancer stem cell inhibitor BBI608 administered with paclitaxel in patients with advanced malignancies (asbtract number 2530). The 2014 American Society of Clinical Oncology (ASCO) Annual Meeting; 30 May-3 June, 2014; Chicago, IL, USA, 2014.
48. Becerra C, Stephenson J, Jonker D, et al., BBl608-201: Phase lb/ II Study of Cancer Stemness Inhibitor BBI608 Combined with Paclitaxel in Advanced Gastric and Gastroesophageal Junction (GE) Adenocarcinoma The 2015 American Society of Clinica Oncology (ASCO) Annual Meeting: 29 May-2 June, 2015: Chicago, IL, USA, 2015.

49. Shah MA MK, Shitra K, Tebbutt NC, et al., The BRIGHTER trial: A phase III randomized, double-blind, placebo-controlled clinical trial of first-in-class cancer stemness inhibitor BBI608 plus weekly paclitaxel versus placebo plus weekly paclitaxel in adult patients with advanced, previously treated gastric and gastroesophageal junction adenocarcinoma. The 2015 American Society of Clinical Oncology (ASCO) Annual Meeting: 29 May-2 June, 2015; Chicago, IL, USA, 2015.

50. Hubbard JM ID, O'Neil BH, Halfdanarson TR, et al., Bl608-246: A Phase Ib study of first-in-class cancer stemness inhibitor BBI608 in combination with FOLFIRI with and without Bevacizumab in Patients with Advanced Colorectal Cancer The American Society of Clinical Oncology (ASCO) 2015 Annual Meeting; 29 May-2 June 2015; Chicago, IL, USA, 2015

51. Ciombor K EW, Hubbard JM, O'Dwyer P, et al., BBI608-224: A Phase $\mathrm{lb} / \mathrm{ll}$ study of cancer stemness inhibitor BBI608 administered with Panitumumab in KRAS wild-type patients with metastatic colorectal cancer The American Society of Clinical Oncology (ASCO) 2015 Annual Meeting; 29 May -2 June 2015; Chicago, IL, USA, 2015

52. Jonker $\mathrm{D}$, Nott, LM, Yoshino, T, et al., The NCIC CTG and AGITG CO.23 trial: A phase III randomized study of BBI608 plus best supportive care (BSC) versus placebo (PBO) plus BSC in patients (Pts) with pretreated advanced colorectal carcinoma (CRC) (abstract no. TPS3660). The 2014 American Society of Clinical Oncology (ASCO) Annual Meeting; 30 May-3 June; Chicago, IL, USA, 2014.

53. Benedito R, Rocha SF, Woeste M, et al., Notch-dependent VEGFR3 upregulation allows angiogenesis without VEGF-VEGFR2 signalling, Nature, 2012;484:110-4

54. Radtke F, Raj K, The role of Notch in tumorigenesis: oncogene or tumour suppressor? Nature Reviews Cancer, 2003:3:756-67.

55. Ranganathan P, Weaver KL, Capobianco AJ, Notch signalling in solid tumours: a little bit of everything but not all the time, Nature Reviews Cancer, 2011:11:338-51.

56. Espinoza I, Pochampally R, Xing F, et al., Notch signaling: targeting cancer stem cells and epithelial-to-mesenchyma transition, Onco Targets Ther, 2013;6:1249-59.

57. Yuan $X, W u H, X u H$, et al., Notch signaling: An emerging therapeutic target for cancer treatment, Cancer Lett, 2015;369:20-7

58. Messersmith WA, Shapiro GI, Cleary JM, et al., A Phase I, dosefinding study in patients with advanced solid malignancies of the oral gamma-secretase inhibitor PF-03084014, Clin Cancer Res, 2015;21:60-7.

59. Valone T, Yeatman TJ, Sullivan D, Phase II study of RO4929097 in metastatic colorectal cancer, J Clin Oncol, 2011;29(suppl):abstract e14058.

60. Lobry C, Oh P, Aifantis I, Oncogenic and tumor suppressor functions of Notch in cancer: it's NOTCH what you think, J EXp Med, 2011:208:1931-5.

61. Andersen P, Uosaki H, Shenje LT, Kwon C, Non-canonical Notch signaling: emerging role and mechanism, Trends Cell Biol, 2012;22:257-65

62. Zhou W, Wang G, Guo S, Regulation of angiogenesis via Notch signaling in breast cancer and cancer stem cells, Biochim Biophys Acta, 2013;1836:304-20

63. Yen WC, Fischer MM, Axelrod F, et al., Targeting Notch signaling with a Notch2/Notch3 antagonist (tarextumab) inhibits tumor growth and decreases tumor-initiating cell frequency, Clin Cancer Res, 2015;21:2084-95

64. Smith DC, Eisenberg PD, Manikhas G, et al., A phase I dose escalation and expansion study of the anticancer stem cell agent demcizumab (anti-DLL4) in patients with previously treated solid tumors, Clin Cancer Res, 2014;20:6295-303.

65. Li Y Welm B, Podsypanina $K$, et al. Evidence that transgenes encoding components of the Wnt signaling pathway preferentially induce mammary cancers from progenitor cells, Proc Natl Acad Sci U S A, 2003:100:15853-8.

66. Boyault S, Rickman DS, de Reynies A, et al., Transcriptome classification of HCC is related to gene alterations and to new. therapeutic targets, Hepatology, 2007:45:42-52.

67. Brabletz T, Jung A, Reu S, et al., Variable beta-catenin expression in colorectal cancers indicates tumor progression driven by the tumor environment, Proc Natl Acad Sci U S A, 2001:98:10356-61.

68. Jonker JJ, Stephenson J, Edenfield WJ, A phase I extension study of BBI608, a first-in-class cancer stem cell (CSC) inhibitor, in patients with advanced solid tumors, J Clin Oncol Res, 2014:32(5s):abstract 2546 .

69. Smith $\mathrm{D}$, Gordon $\mathrm{M}$, Messersmith W, A first-in-human phase I study of anti-cancer stem cell (CSC) agent OMP-54F28 (FZD8-FC) targeting the Wnt pathway in patients with advanced solid tumors [abstract], Mol Cancer Ther, 2013;12(Suppl 11):B79.

70. Schaller MD, Borgman CA, Cobb BS, et al., pp125FAK a structurally distinctive protein-tyrosine kinase associated with focal adhesions, Proc Natl Acad Sci U S A, 1992;89:5192-6.

71. Luo M, Fan H, Nagy T, et al., Mammary epithelial-specific ablation of the focal adhesion kinase suppresses mammary tumorigenesis by affecting mammary cancer stem/progenito cells, Cancer Res, 2009;69:466-74.

72. Schober M, Fuchs E, Tumor-initiating stem cells of squamous cell carcinomas and their control by TGF-beta and integrin/ focal adhesion kinase (FAK) signaling, Proc Natl Acad Sci U S A, 2011:108:10544-9.

73. Ho B, Olson G, Figel $\mathrm{S}$, et al., Nanog increases focal adhesion kinase (FAK) promoter activity and expression and directly binds to FAK protein to be phosphorylated, I Biol Chem, 2012;287:18656-73

74. Peng $\mathrm{X}$, Ueda $\mathrm{H}$, Zhou $\mathrm{H}$, et al., Overexpression of focal adhesion kinase in vascular endothelial cells promotes angiogenesis in transgenic mice, Cardiovasc Res, 2004:64:421-30.

75. Scheel C, Weinberg RA, Phenotypic plasticity and epithelialmesenchymal transitions in cancer and normal stem cells? Int Cancer, 2011:129:2310-4.

76. Fan $\mathrm{C}$, Oh DS, Wessels $\mathrm{L}$, et al., Concordance among geneexpression-based predictors for breast cancer, N Engl J Med 2006;355:560-9.

77. Golubovskaya VM, Targeting FAK in human cancer: from finding to first clinical trials, Front Biosci (Landmark Ed), 2014:19:687-706.

78. Massague J, Blain SW, Lo RS, TGFbeta signaling in growth control, cancer, and heritable disorders, Cell, 2000:103:295-309.

79. Chang $\mathrm{H}$, Brown $\mathrm{CW}$, Matzuk MM, Genetic analysis of the mammalian transforming growth factor-beta superfamily, Endocr Rev, 2002;23:787-823.

80. Taipale J, Beachy PA, The Hedgehog and Wnt signalling pathways in cancer, Nature, 2001;411:349-54.

81. Sicklick JK, Li YX, Jayaraman A, et al., Dysregulation of the Hedgehog pathway in human hepatocarcinogenesis, Carcinogenesis, 2006:27:748-57.

82. Coni S, Infante $\mathrm{P}$, Gulino A, Control of stem cells and cancer stem cells by Hedgehog signaling: pharmacologic clues from pathway dissection, Biochem Pharmacol, 2013;85:623-8.

83. Tanaka S, Cancer stem cells as therapeutic targets of hepatobiliary-pancreatic cancers, I Hepatobiliary Pancreat Sci, 2015;22:531-7.

84. Cohen DJ, Christos PJ, Kindler HL, et al., Vismodegib (V), a hedgehog $(\mathrm{HH})$ pathway inhibitor, combined with FOLFOX for first-line therapy of patients (pts) with advanced gastric and gastroesophageal junction (GEJ) carcinoma: A New York Cancer Consortium led phase II randomized study, J Clin Oncol, 2013;31(Suppl):abstract 4011.

85. He XC, Yin T, Grindley JC, et al., PTEN-deficient intestinal stem cells initiate intestinal polyposis, Nat Genet, 2007:39(2):189-98.

86. Kangwan N, Park JM, Kim EH, Hahm KB, Chemoquiescence for ideal cancer treatment and prevention: where are we now? Eur J Cancer Prev, 2014:19:89-6.

87. Kimura T, Takabatake Y, Takahashi A, Isaka Y, Chloroquine in cancer therapy: a double-edged sword of autophagy, Cancer Res, 2013;73:3-7.

88. Solomon VR, Lee $\mathrm{H}$, Chloroquine and its analogs: a new promise of an old drug for effective and safe cancer therapies, Eur Pharmacol, 2009;625:220-33.

89. Maycotte $\mathrm{P}$, Aryal $\mathrm{S}$, Cummings $\mathrm{CT}$, et al., Chloroquine sensitizes breast cancer cells to chemotherapy independent of autophagy, Autophagy, 2012;8:200-12.

90. Wolchok JD, Hoos A, O'Day S, et al., Guidelines for the evaluation of immune therapy activity in solid tumors: immunerelated response criteria, Clin Cancer Res, 2009;15:7412-20.

91. Wang ML, Chiou SH, Wu CW, Targeting cancer stem cells: emerging role of Nanog transcription factor, Onco Targets Ther, 2013:6:1207-20. 